\title{
Watermelon chlorotic stunt virus from the Sudan and Iran: Sequence Comparisons and Identification of a Whitefly-Transmission Determinant
}

\author{
A. Kheyr-Pour, K. Bananej, G. A. Dafalla, P. Caciagli, E. Noris, A. Ahoonmanesh, H. Lecoq, and B. Gronenborn
}

First, second, and eighth authors: Institut des Sciences Végétales, CNRS, 91198 Gif-sur-Yvette, France; second author: Department of Plant Pathology, College of Agriculture, Tarbiat Modarres University, Tehran, Iran; third author: Plant Pathology Center, Faculty of Agriculture, University of Gezira, Wad Medani, Sudan; fourth and fifth authors: Istituto di Fitovirologia Applicata, CNR, 10135 Torino, Italy; sixth author: Plant Protection Department, Faculty of Agriculture, Isfahan University of Technology, Isfahan, Iran; and seventh author: Station de Pathologie Végétale, INRA, 84143 Montfavet, France.

Accepted for publication 15 February 2000.

\begin{abstract}
Kheyr-Pour, A., Bananej, K., Dafalla, G. A., Caciagli, P., Noris, E., Ahoonmanesh, A., Lecoq, H., and Gronenborn, B. 2000. Watermelon chlorotic stunt virus from the Sudan and Iran: Sequence comparisons and identification of a whitefly-transmission determinant. Phytopathology 90: 629-635.

The genomes of two Watermelon chlorotic stunt virus (WmCSV) isolates, one from the Sudan and one from Iran, were cloned and sequenced. Sequence relationship with other geminiviruses characterizes WmCSV as

a typical Eastern Hemisphere geminivirus with a bipartite genome. The two geographically distant WmCSV isolates from Africa and the Middle East share a very high overall sequence similarity: $98 \%$ between their DNA-A and $96 \%$ between their DNA-B components, and their respective capsid proteins are identical. A single amino acid change in the capsid protein (N131D) renders WmCSV whitefly nontransmissible. This region of the capsid is also implicated in transmission by Bemisia tabaci of Tomato yellow leaf curl virus.
\end{abstract}

A whitefly-transmitted disease of watermelon had been reported from Yemen and its causal agent was described as Watermelon chlorotic stunt virus (WmCSV), a member of the geminivirus family $(5,54)$.

Members of the family Geminiviridae are plant pathogens with circular, single-stranded DNA genomes encapsidated in twin particles comprising the genera Mastrevirus, Curtovirus, and Begomovirus (41). They are differentiated based upon genome organization and insect vector specificity $(20,29)$. Most begomoviruses have a bipartite genome that consists of a DNA-A and a DNA-B component. Notable exceptions are the tomato (yellow) leaf curl viruses from the Near East, the Mediterranean Basin, Australia, and India whose genome is monopartite $(14,27,35)$ (GenBank no. U38293). Irrespective of their genome size and segmentation, all begomoviruses are transmitted by the whitefly Bemisia tabaci (Gennadius).

Between 1993 and 1996, severe outbreaks of a whitefly-transmitted disease occurred in cucurbit-growing regions of the central and eastern Sudan, affecting both watermelon (Citrullus lanatus (Thunb.) Matsum. \& Nakai) and melon (Cucumis melo L.) (12, 30). Also, the watermelon and melon cultures of the southern provinces of Iran (Bushehr, Hormouzgan, and SistanBalouchistan) were severely infected with a whiteflyassociated virus, and the plants had symptoms similar to those described for WmCSV (4).

In order to identify and further characterize the pathogen causing the watermelon and melon diseases in the Sudan and in south-

\section{Corresponding author: B. Gronenborn; E-mail address: gronenborn@isv.cnrs-gif.fr}

The DNA sequences of Watermelon chlorotic stunt virus from the Sudan and Iran have been deposited in the EMBL, GenBank, and DDBJ databases under accession numbers AJ245650, AJ245651, AJ245652, and AJ245653.

Publication no. P-2000-0418-02R

(C) 2000 The American Phytopathological Society ern Iran, samples were analyzed by electron microscopy, serology, and DNA hybridization. Here, we describe the molecular characterization of WmCSV, an Eastern Hemisphere geminivirus with a bipartite genome. Furthermore, transmission assays by $B$. tabaci of the virions derived from cloned DNA in combination with targeted mutagenesis revealed a molecular determinant in the viral capsid protein essential for whitefly transmissibility.

\section{MATERIALS AND METHODS}

Field surveys for WmCSV. Surveys of plants suspected to be infected by WmCSV were conducted in the fall and winter seasons of 1993 through 1997 in different cucurbit-growing regions of the Sudan: the Khartoum area, Kassala in the east, Damazin in the south, and El Obeid in the west. From each location, 2 to 12 samples of cultivated or wild cucurbits showing a range of mosaic, yellowing, stunting, leaf and fruit discoloration, and deformation symptoms were collected. WmCSV was detected by double-antibody sandwich (DAS) enzyme-linked immunosorbent assay (ELISA) using polyclonal antisera against WmCSV from Yemen (54) and the Sudan. As soon as cloned copies of the viral genome were available, dot- and squash-blots of plant sap on nylon membranes were mainly used (34). Similarly, melon and watermelon fields in the southern provinces of Iran (Bushehr, Hormouzgan, and SistanBalouchistan) were surveyed in 1997 and 1998 by leaf blotting, and virus-infected samples were collected.

Cloning of the WmCSV genome from the Sudan and Iran. Standard techniques of molecular biology were as described by Ausubel et al. (3).

Replicative forms of geminivirus DNA were prepared from WmCSV-infected plants from Wad Medani (Sudan) and from Bandar Abbas (Iran) according to Bendahmane et al. (6). Polymerase chain reaction (PCR) amplification (23) was similar to the method described by Rybicki and Hughes (45), with consensus primers designed to amplify whitefly-transmitted geminiviruses (Table 1). 
Restriction endonucleases that cleaved the amplified DNA only once were used to clone full-length genomic DNAs of WmCSV from total DNA preparations, using the amplified DNA, labeled by ${ }^{32} \mathrm{P}$, as a probe. WmCSV DNA-A was linearized by HindIII and inserted into the corresponding site of pUC118 (pWmCSV-AHind-11) as described by Kheyr-Pour et al. (27). Three independent clones of WmCSV DNA-B were constructed in the XbaI site and two in the PstI site of pUC118. One DNA-A clone (pWmCSV-AHind-11) and one DNA-B clone (pWmCSV-B-Xba-59) were used for infectivity assays by agroinoculation, and the sequences of the WmCSV DNAs were determined.

To clone the DNA-A of WmCSV from Iran, a PCR-amplified fragment by primers WTG-BsiWI-(v) and WTG-BsiWI-(c) (Table 1) was partially sequenced, and two complementary oligonucleotide primers overlapping a unique $X b a \mathrm{I}$ site were used to amplify and clone the full-length DNA-A of WmCSV-Ir in pUC118 (pWmCSVA-Xba-28). Similarly, a DNA-B fragment was amplified and partially sequenced, and a complete DNA-B copy was cloned via a unique SmaI site in pUC118 (pWmCSV-B-Sma-23).

DNA sequence determination. The DNA sequences of the cloned WmCSV DNAs were determined by cycle sequencing using the fluorescent dye dideoxy chain terminator technology and an Applied Biosystems 373A sequencer (Perkin-Elmer Applied Biosystems, Corp., Foster City, CA) (53). Sets of nested-deletion mutants between restriction sites in the WmCSV DNA and the polylinker of pUC118 were constructed and served as sequencing templates. Regions of the genome whose sequence could not be determined this way by the universal and reverse sequencing primers were subcloned or were sequenced using synthetic primers. The DNA sequence of both strands was covered by several independent reads, and the UWGCG sequence analysis package was used to assemble and analyze the sequences (13).

Construction of WmCSV mutants. Site-directed mutagenesis was performed using synthetic oligonucleotides and the Quikchange mutagenesis kit (Stratagene Inc., La Jolla, CA). The respective mutagenic primers are listed in Table 1 . The mutations introduced were identified by restriction endonuclease digests (where possible) and confirmed by sequencing.
To convert the whitefly-nontransmissible WmCSV into a transmissible virus, primers AV1-D131N-(v/c) (Table 1) were used to change nucleotide 706 (G) in DNA-A of WmCSV-Sd into A. This change alters aspartate 131 of the capsid protein to asparagine $(\mathrm{D} 131 \mathrm{~N})$. The change was introduced into plasmid pWmCSV-AHind-11 and identified by sequencing.

Open reading frame (ORF) AC4 of WmCSV terminates early after amino acid 47 by a TGA codon. To suppress the termination codon, two complementary oligonucleotides (Table 1) were used to alter $\mathrm{G} 2330$ into $\mathrm{C}$, creating a serine codon at position 48 of ORF AC4 in plasmid pWmCSV-A-Hind-11. The nucleotide change introduced an AatII restriction site; sequencing confirmed its correctness.

A triple-termination mutation in the putative ORF AC5 was created by PCR-based mutagenesis directly on plasmid pWmCSVA-Hind-11. Three pairs of oligonucleotides (Table 1) spanning the codons R32, G76 (suppressing an NdeI site), and R146 (suppressing a $B s i$ WI site) of AC5, and altering each of them into a TGA codon, were used. All changes were confirmed by sequencing.

DNA-B of WmCSV from the Sudan has an uncommon ORF, BV2. It was mutated by filling in a unique $X h o I$ site with a Klenow fragment of polymerase I and subsequent ligation of plasmid pWmCSV$\mathrm{B}-\mathrm{Xba}-59$. The correct addition of four nucleotides in ORF BV2 was verified by sequencing. The thus altered ORF BV2 terminates prematurely after a further 37 fortuitous codons.

Agroinoculation assays. Full-length WmCSV DNA-A and DNA-B inserts were released from pUC118 by the restriction enzymes that had been used for cloning. Dimers of the respective WmCSV DNAs were inserted into the binary T-DNA vector pBin19 (7). The resulting plasmids were transferred into Agrobacterium tumefaciens strain LBA 4404 by electroporation and used for agroinoculation as described (27). Two watermelon cultivars (Citrullus lanatus cvs. Sugar Baby and Crimson Sweet), melon (Cucumis melo cv. Ananas, and a cultivar from Meshed, Iran), and Nicotiana benthamiana were inoculated as 3- to 4-week-old plants. Symptoms characteristic of the disease developed at about 2 weeks postinoculation.

Whitefly transmission experiments. $B$. tabaci, biotype $\mathrm{B}$, were reared and handled as described (8). For virus transmission, insects

TABLE 1. Oligonucleotides used for genome amplification and mutagenesis by polymerase chain reaction

Primer pairs ${ }^{\mathrm{a}}$;

genome positions (nt)

Sequences

Description and purpose

WTG-BsiWI-(v): 542-566

WTG-BsiWI-(c): 550-520

WAI-XbaI-(v): 1,676-1,700

WAI-XbaI-(c): 1,693-1,669

WAI-SmaI-(v): 1,410-1,434 WAI-SmaI-(c): 1,427-1,402

AV1-D131N-(v): 697-723 AV1-D131N-(c): 723-697

AC4*48S-(v): 2,315-2,350 AC4*48S-(c): 2,350-2,315

AC5-R32*-(v): 880-906

AC5-R32*-(c): 906-880

AC5-G76*-(v): 748-774

AC5-G76*-(c): 774-748

AC5-R146*-(v): 533-557

AC5-R146*-(c): 557-533 $5^{\prime}$-a(ag)tcgtacga(ga)ca(ag)cg(agct)(cg)atga(tc)(ag)t-3

$5^{\prime}$-gggcgtacga(tc)tg(ag)ac(tc)ttaa(agt)ga(acgt)ccttcaca-3'

$5^{\prime}$-tcctcctctagaaactctttatacg-3'

$5^{\prime}$-agagtttctagaggaggagaagaac- $3^{\prime}$

$5^{\prime}$-gagtctcccgggtctagcgtggctg- $3^{\prime}$

$5^{\prime}$-gctagacccgggagactcagtcttcc-3'

$5^{\prime}$-aaaaaacaaaatcatacgaatcaggtc- $3^{\prime}$

$5^{\prime}$-gacctgattcgtatgat tttgtttttt-3'

$5^{\prime}$-tccaggtaagacttgacgtctgtactgacttagc- $3^{\prime}$

$5^{\prime}$-gctaagtccagtacagacgt caagtcttacctgg-3'

$5^{\prime}$-gttggtggcccetcagggatgaaggag- $3^{\prime}$

$5^{\prime}$-ctccttcatcc tgaggggccaccaac- $3^{\prime}$

$5^{\prime}$-cgtccatacgggtcaagtccaatggac- $3^{\prime}$

$5^{\prime}$-gtccattggacttgaccegtatggacgacg-3'

$5^{\prime}$-gcaaagttcagtc atacgaacaacg-3'

$5^{\prime}$-cgttgttcgtatgactgaactttgc-3'
Consensus primers used to amplify full-length DNA-A of whitefly-transmitted geminiviruses ${ }^{b}$

Consensus primers used to amplify full-length DNA-A of whitefly-transmitted geminiviruses ${ }^{b}$

Used to amplify full-length DNA-A of WmCSV from $\operatorname{Iran}^{\mathrm{c}}$ Used to amplify full-length DNA-A of WmCSV from Iran ${ }^{c}$

Used to amplify full-length DNA-B of WmCSV from Iran ${ }^{\mathrm{d}}$ Used to amplify full-length DNA-B of WmCSV from Iran ${ }^{\mathrm{d}}$

Change G706 to A in the capsid D131N mutant Change G706 to $A$ in the capsid D131N mutant

Change C2330 to $\mathrm{G}$, alter codon 48 (TGA) of AC4 to TCA Change C2330 to $\mathrm{G}$, alter codon 48 (TGA) of AC4 to TCA

Change T894 to A, generate a TGA codon at Arg 32 of AC5 Change T894 to A, generate a TGA codon at Arg 32 of AC5

Change C762 to A, generate a TGA at Gly 76 of AC5; the T756-to-C change suppresses a NdeI site

Change C762 to A, generate a TGA at Gly 76 of AC5; the T756-to-C change suppresses a NdeI site

Change G546 to A, generate a TGA at Arg 146 of AC5, and suppress a $B$ siWI site

Change G546 to A, generate a TGA at Arg 146 of AC5, and suppress a $B$ siWI site

$\mathrm{a}(\mathrm{v})=$ Virion-sense polarity, and $(\mathrm{c})$ = complementary-sense polarity.

${ }^{\mathrm{b}}$ BsiWI site is underlined; nonviral bases in italics; coordinates refer to DNA-A of Watermelon chlorotic stunt virus, Sudan isolate (WmCSV-Sd).

c XbaI site is underlined.

d SmaI site is underlined. 
were allowed to feed for $48 \mathrm{~h}$ on watermelon plants cv. Sugar Baby infected by agroinoculation. Subsequently, groups of 15 insects were moved to single watermelon plants of the same cultivar for transmission. Three days later, insects were removed and plants were sprayed with an insecticide mixture of $0.15 \%$ acephate (Orthene) and $0.2 \%$ buprofezin (Applaud) (SIPCAM SpA, Milano, Italy) and maintained in a whitefly-free greenhouse. Six to eight weeks after inoculation, individual plants were assayed for the presence of viral DNA by leaf squash blotting.

Sequence comparison. DNA and protein sequences were compared using the algorithms provided by the UWGCG sequence analysis package (version 10.1; Genetic Computer Group, Inc., Madison, WI). Multiple sequence alignments were done by PileUp (13) or Clustal W (51) using default parameters. Trees were generated and displayed by PAUP (UWGCG version) or PAUP* 4.0 beta version 2a (PPC) (D. L. Swofford, Illinois Natural History Survey, Champaign, IL) using 1,000 bootstrap repeats and a cutoff value of $60 \%$.

Begomoviruses used for DNA-A genome comparisons. Abbreviations of virus names and isolates are according to Fauquet and Mayo (17). Database numbers follow the virus names.

Western Hemisphere begomoviruses include Abutilon mosaic virus (AbMV), X15983; Bean dwarf mosaic virus (BDMV), M88179; Bean golden mosaic virus, Brazil isolate (BGMV-Br), M88686; Bean golden mosaic virus, Puerto Rico isolate (BGMV-Pr), M10070; Pepper huasteco virus (PHV), X70418; Potato yellow mosaic virus (PYMV), D00940; Squash leaf curl virus (SLCV), M38183; Tomato golden mosaic virus (TGMV), K02029; Tomato mottle virus, Florida isolate (ToMoV), L14460; and Taino tomato mottle virus, Cuba isolate (TToMoV), AF012300.

Eastern Hemisphere begomoviruses include African cassava mosaic virus, Kenya isolate (ACMV-Ke), J02057; African cassava mosaic virus, Nigeria isolate (ACMV-Ni), X17095; Althea rosea enation virus (AREV), AF014881; Angled luffa leaf curl virus, Thailand isolate (AnLCV-Th), AF102276; Ageratum yellow vein virus (AYVV), X74516; Cotton leaf curl virus, Pakistan isolate clc62 (CLCuV-Pk1), AJ002447; East African cassava mosaic virus, Tanzania isolate (EACMV-Tz), Z83256; Indian cassava mosaic virus (ICMV), Z24758; Mungbean yellow mosaic virus (MYMV), D14703; Squash leaf curl virus, China isolate (SLCV-Cn), AB027465; Tomato leaf curl virus, Australia isolate (ToLCV-Au), S53251; Tomato leaf curl virus, New Delhi 1 isolate (ToLCV-NDe1), U15015; Tomato leaf curl virus, Bangalore II isolate (ToLCV-BanII), U38239; Tomato yellow leaf curl virus, Iran isolate (TYLCV-Ir), AJ132711; Tomato yellow leaf curl virus, Israel isolate (TYLCV-Il), X15656; Tomato yellow leaf curl virus, Sardinia isolate (TYLCV-Sar), X61153;

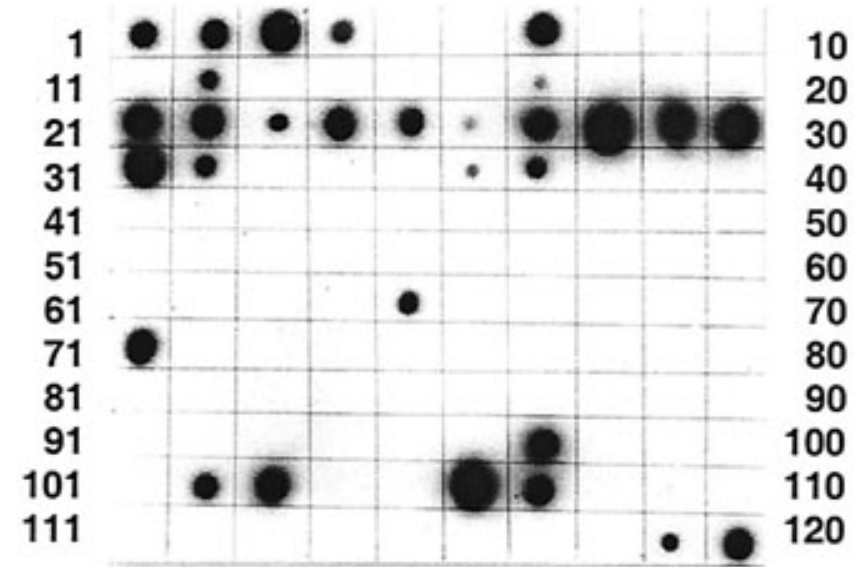

Fig. 1. Dot-blot hybridization of multiple samples from a Watermelon chlorotic stunt virus (WmCSV) field survey in the Sudan in 1994. About $3 \mu \mathrm{l}$ of plant sap was applied to the nylon membrane. ${ }^{32} \mathrm{P}$-labeled WmCSV DNA-A was used as a probe. The intensity of the spots is proportional to the amount of viral nucleic acid present in the samples. No. 120 contains $3 \mu$ of plant sap of a WmCSV-infected laboratory control plant.
Tomato yellow leaf curl virus, Thailand 1 isolate (TYLCV-Th1), $\mathrm{X} 63015$; Watermelon chlorotic stunt virus, Iran isolate (WmCSVIr), AJ245652; and Watermelon chlorotic stunt virus, Sudan isolate (WmCSV-Sd), AJ245650.

The curtovirus Beet curly top virus (BCTV-CFH) (U02311) is used as an outgroup in the phylogenetic trees.

\section{RESULTS AND DISCUSSION}

Field survey. A comparative survey among samples of diseased cucurbits from the Sudan, collected in 1994, was carried out by DAS-ELISA and dot-blot hybridization. Extracts of 120 samples from cultivated watermelon, melon, snake cucumber (Cucumis melo var. flexuosus), and squash (Cucurbita moschata (Duchesne) Duchesne ex Poir.), and from wild Cucumis melo var. agrestis plants were spotted on a nylon membrane and hybridized with a ${ }^{32}$ P-labeled DNA-A probe (Fig. 1). All samples that reacted positively in DAS-ELISA also reacted with the labeled probe. The intensity of the hybridization signal correlated with the amount of single-stranded viral DNA in the tested material. The 29 positive samples included 14 watermelon, 7 melon, 7 snake cucumber, and 1 wild melon (No. 12). Amounts of viral DNA varied among samples, and no host-specific variation in the virus titer was apparent. Additional field observations in the Sudan during 1995 to 1997 confirmed that WmCSV causes considerable crop losses in extensive commercial melon cultures (13).

WmCSV DNA also was detected in blotted samples from cucumber, squash, and wild watermelon (Citrullus colocynthis) collected in the Bandar Abbas area (southern Iran), indicating that the virus was present in most cultivated cucurbits tested. Numerous tomato samples collected in the Sudan did not contain WmCSV, and repeated agroinoculation assays of tomato by cloned WmCSV DNA-A and DNA-B failed to cause any infection (12). Apart from the permissive host $N$. benthamiana, only cucurbits have been found to be a host of WmCSV.

Agroinfectious clones of WmCSV. By applying degenerate consensus primers (Table 1), we amplified the DNA-A of WmCSV and used it as a probe for cloning the complete DNA-A of the virus isolates from central Sudan and southern Iran. Specific probes of the common region in conjunction with diagnostic restriction endonuclease digests served to identify and clone the respective DNA-B components of the two isolates.

Complete genomic clones of WmCSV from both the Sudan (pWmCSV-A-Hind-11, pWmCSV-B-Xba-59) and from Iran (pWmCSV-A-Xba-28, pWmCSV-B-Sma-23) were sequenced (Fig. 2; Table 2). Their infectivity and bipartite nature were proven by agroinoculation of watermelon, melon, and $N$. benthamiana (Table 3).

Genome organization and sequence comparison. The DNAAs of the WmCSV isolates from the Sudan and from Iran consisted of 2,753 or 2,752 nucleotides (nt), respectively. DNA-B of

TABLE 2. Open reading frames (ORFs) of Watermelon chlorotic stunt virus (WmCSV)

\begin{tabular}{|c|c|c|c|c|c|}
\hline \multirow[b]{2}{*}{ ORF } & \multicolumn{2}{|c|}{ Sequence coordinates $^{\mathrm{a}}$ (nucleotides) } & \multirow{2}{*}{$\begin{array}{c}\text { Amino } \\
\text { acids }\end{array}$} & \multicolumn{2}{|c|}{ Deduced $\mathrm{MW}^{\mathrm{b}}(\mathrm{kDa})$} \\
\hline & WmCSV-Sd & WmCSV-Ir & & WmCSV-Sd & WmCSV-Ir \\
\hline AV2 & $156-515$ & $155-514$ & 119 & 13.2 & 13.7 \\
\hline AV1 & $316-1,092$ & $315-1,091$ & 258 & 30.1 & 30.1 \\
\hline $\mathrm{AC} 1$ & $2,626-1,541$ & $2,625-1,540$ & 361 & 41.1 & 41.1 \\
\hline $\mathrm{AC} 2$ & $1,641-1,234$ & $1,640-1,233$ & 135 & 15.5 & 15.6 \\
\hline AC3 & $1,493-1,089$ & $1,492-1,088$ & 134 & 15.8 & 15.9 \\
\hline $\mathrm{AC} 4$ & $2,472-2,329$ & $2,471-2,328$ & 47 & 4.9 & 4.9 \\
\hline AC5 & $987-220$ & $986-219$ & 255 & 28.2 & 28.2 \\
\hline BV2 & $220-531$ & Absent & 103 & 11.6 & Absent \\
\hline BV1 & $531-1,286$ & $500-1,255$ & 251 & 28.9 & 28.8 \\
\hline $\mathrm{BC} 1$ & $2,246-1,326$ & $2,215-1,295$ & 306 & 34.8 & 34.7 \\
\hline
\end{tabular}

a Sequence numbering begins at base eight (A) of the conserved nonanucleotide TAATATT ${ }^{1}$ AC, according to Laufs et al. (28). Sudan isolate $=-\mathrm{Sd}$, and Iran isolate $=-$ Ir.

b $\mathrm{MW}=$ molecular weight. 
WmCSV-Sd contains 2,760 nt, whereas DNA-B of WmCSV-Ir is slightly smaller $(2,728 \mathrm{nt})$. Sequence numbering begins at base eight (A) of the conserved nonanucleotide TAATATT ${ }^{1} \mathrm{AC}$, the first base of viral (+)-strand DNA synthesized after replication initiation by the Rep protein (28). A short stretch of 30 bases (nt 69 to 99) is missing in the right part of the intergenic region of DNA-B of the Iran isolate (Fig. 3). The overall sequence identity between the Sudan and Iran isolates is $98.3 \%$ for DNA-A and $95.5 \%$ for DNA-B. The common region has a length of 131 bases with 22 base differences. It has the typical inverted repeat sequence (hairpin) including the conserved nonanucleotide. Of particular interest is a one-nucleotide difference in the stem sequence of the hairpin between DNA-A and DNA-B, found in both WmCSV isolates. The sequence in the stem of DNA-B (GGCC) is the same as in all other whitefly-transmitted geminiviruses sequences available in the databases, whereas DNA-A has the sequence GCCC at this position (Fig. 3). Hence, WmCSV represents a natural example of hairpin polymorphism between DNA-A and DNA-B, similar to the artificial replication proficient hairpin mutants constructed by Orozco and Hanley-Bowdoin (37).

Three direct repeat sequences of seven bases (iterons) are located at the $5^{\prime}$ end of the hairpin sequence in the left part of the common region (Fig. 3). These iterons are the presumed recognition sites of Rep protein $(1,2,11,18)$. The sequence identity of the iterons between the two WmCSV isolates is in accordance with the complete conservation of the amino acid sequence of the respective WmCSV Rep protein domains implicated in specific origin recognition $(9,10,25)$. There are only four amino acid differences
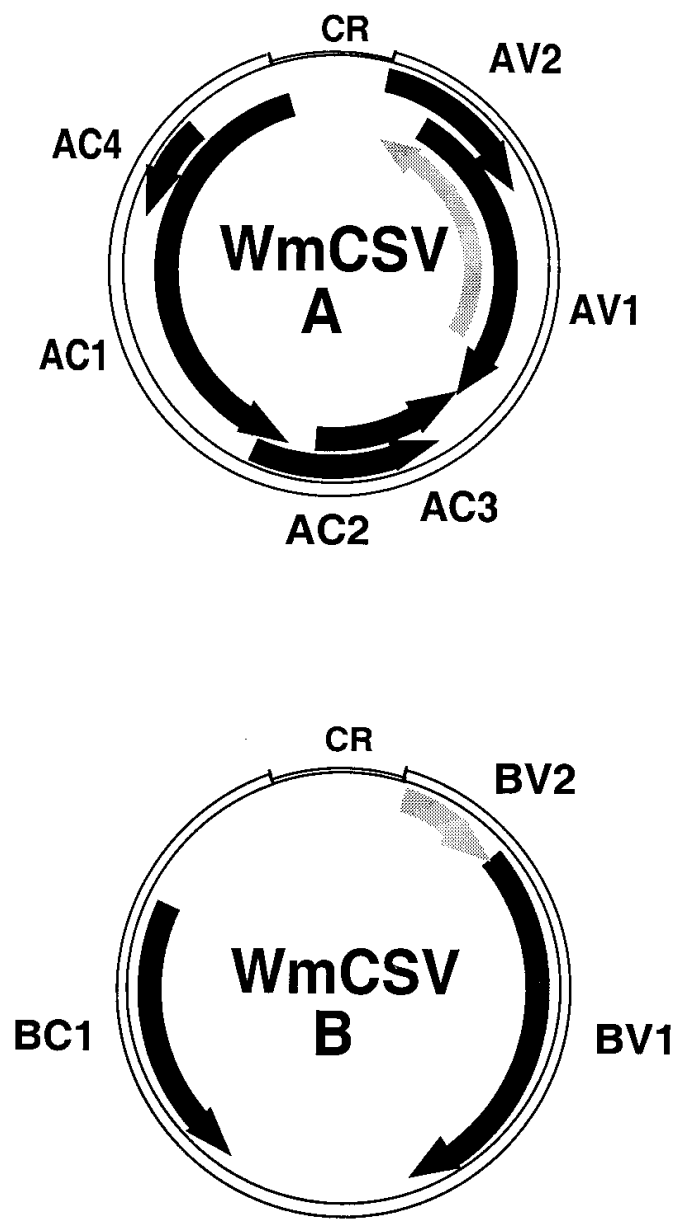

Fig. 2. The bipartite genome of Watermelon chlorotic stunt virus (WmCSV). Arrows represent genes: $\mathrm{V}$ denotes virion-sense and $\mathrm{C}$ denotes complementarysense polarity. CR indicates the common region of DNA-A and DNA-B. Gray arrows represent the dispensable open reading frames AC5 and BV2; the latter is present only in WmCSV-Sd. between the Rep protein of WmCSV-Sd and WmCSV-Ir, M190L, D197E, T336S, and T342A, all located in the central or carboxyterminal part of the protein, outside its origin recognition domain.

A partial sequence of WmCSV from Yemen, 410 bases of the rep gene (GenBank no. X79430), is slightly closer to the sequence of WmCSV-Ir (98\% identity) than to that of WmCSV-Sd (96\%).

Capsid proteins of WmCSV from the Sudan and Iran are identical. Additional sequence analysis of an independent DNAA clone, constructed using DNA isolated directly from field-infected watermelon plants originating from Wad Medani, Sudan, the same location where the graft-propagated virus was isolated, revealed an asparagine encoded at amino acid position 131. This strongly suggested that, originally, the capsid gene had coded for a protein leading to a whitefly-transmissible virus. Therefore, the capsid protein sequences of the Sudan and Iran WmCSV isolates are considered identical. This is in contrast to other whitefly-transmitted geminiviruses whose capsid protein sequences usually differing by a few percent within a species. For instance, those of TYLCV isolates from Sardinia, Sicily, and Spain, as well as the ones from the Near East, differ by about 1 to $2 \%$.

Multiple pairwise comparisons of the WmCSV DNA-A nucleotide sequence with that of other whitefly-transmitted geminiviruses currently available in the databases characterized the two WmCSV isolates as typical Eastern Hemisphere geminiviruses $(38,44)$. Figure 4 illustrates the relationship of WmCSV DNA-A in the form of a dendrogram. DNA-A sequences of selected Begomovirus species and isolates thereof were aligned using Clustal W (51), and the dendrogram was generated by PAUP (D. L. Swofford,

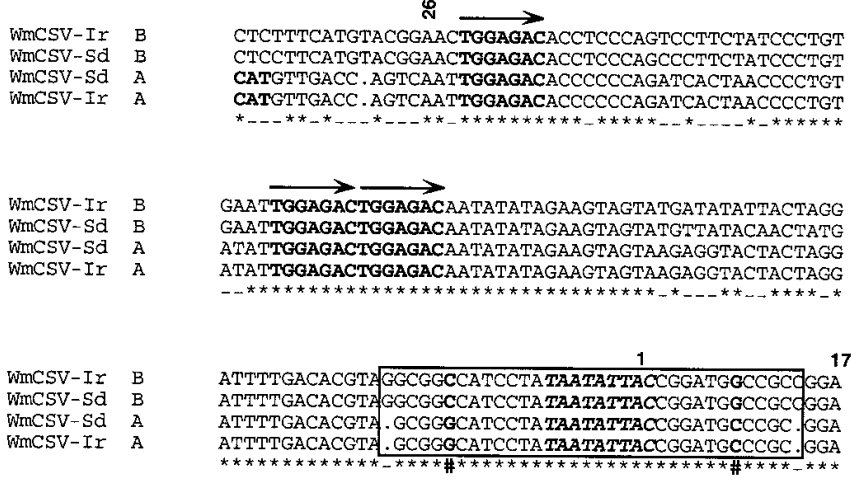

WimcSV-Ir $B$ WinCSV-Sd $\quad B$ WimCSV-Sd A

TCCCATTTGTGCGGACCCCACCGA . . . GTCTCCAAATGGCATTITGGTA GAAAAAAAGG. TGACCCCACAA.....GTCTCCAAATGGCATITTGGTA GCCAAAAAG . TGACCCACAGGACACGHGCACCA TGAAATTGCGTGC GCCAAAAAG ... TGACCCCACAGGACACGTGCACCAATGAAATTGCGTGC

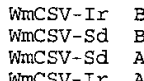

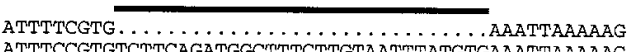
ATTTCCGTGTCTTCAGATGGCTTCTTGTAATTTATGTGAAATTAAAAAG TTTGAGGTAAGT. AGCTGGAAATGGGT . . . . . TTTGAAAGCGACAAG TTTGAGGTAAGTT. AGCTGGAAATGGAGT . . . . . . TTTGAAAGCGACAAG

WhCSV-Ir B WmCSV-Sd $B$ WimCSV-Ir A

AGGGACCAC. CGCACTATCACTCTCTTGGCCAATCAAAGTGCGTCCTTATI AGGGACCAC. CGCACTATCACCTTCTTGGCCAATCAAAGTGCGCCTTGAT

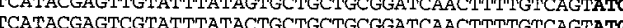
TCATACGAGTCGTATTTATACTGCTGCTGCGGATCAACTTTTGTCAGTATG

Fig. 3. The intergenic and common region of DNA-A and DNA-B. Sequence coordinates refer to DNA-A of Watermelon chlorotic stunt virus, Sudan isolate (WmCSV-Sd). The complete intergenic region of DNA-A of the Sudan and Iran isolates is shown, flanked by the AC1 (CAT) and AV2 (ATG) initiation codons. The common region extends from base 2,639 through base 17. The hairpin sequences with the nonanucleotide (in bold) are framed, and base one (1) of the sequences, as defined by Laufs et al. (28), is marked. The difference in the stem sequences between DNA-As and DNA-Bs is indicated by a \# on the consensus line, $\mathrm{a}^{\wedge}$ points to the one-nucleotide-length difference between DNA-As. A bar marks the sequence present only in DNA-B of WmCSVSd. Iteron sequences are shown in bold and are marked by horizontal arrows. 
Illinois Natural History Survey). WmCSV groups together with the viruses from Africa, the Near East, and the Mediterranean Basin and with the tomato yellow leaf curl viruses from the Sudan and Iran, with the African cassava geminiviruses being its nearest neighbors. The same relationship holds when the deduced protein sequences of Rep (AC1), AV1, AC2, and AC3 ORFs served as the basis for comparison (data not shown).

The high degree of overall DNA sequence conservation between the two geographic isolates of WmCSV is similar to that of the Nigerian and Kenyan isolates of ACMV, which differ by $3.6 \%$ between their DNA-A and 6.2\% between their DNA-B $(33,48)$. By contrast, isolates of EACMV from Eastern Kenya, Malawi, or Tanzania are more divergent and differ by 13 to $14 \%$ in sequence (56).

The reason for the sequence conservation between the WmCSV isolates from the Sudan and Iran remains unclear. In some way, it resembles the cases in which TYLCVs from the Near East were detected in the Caribbean Basin (46), Spain and Portugal (GenBank no. AF071228), or Japan (26). Whereas human introduction of infected plant material appears as the cause for the recent occurrence of these TYLCV species in new and distant geographical locations, the reason for the similarity between the WmCSV isolates from the Sudan and Iran cannot be attributed with certainty to human dissemination. In that respect, it is interesting to note that two TYLCV isolates from the same respective locations in the Sudan and Iran differ by $11.7 \%$ in their DNA sequence (GenBank no. AJ132711) (B. Gronenborn, unpublished data), significantly contrasting the $98 \%$ DNA-A sequence identity of the two WmCSV isolates.

Two other Eastern Hemisphere begomoviruses infecting cucurbits, SLCV-Cn and AnLCV, are geminivirus species distinct from WmCSV; they are part of the Asian cluster of begomoviruses (Fig. 4).

Three additional (partial) sequences of geminiviruses isolated from watermelon are available in the databases (AF070922, AF065820, and AF065819), one from Nicaragua and two from Texas. The deduced 177 amino acids of the capsid protein identify them as Western Hemisphere squash leaf curl viruses (data not shown).

Atypical ORFs of WmCSV. A longer AC4 ORF does not compensate for the requirement of a DNA-B. Similar to ToLCV from India (ToLCV-NDe1/2) (39) and PHV (52) and unlike the monopartite tomato (yellow) leaf curl viruses, WmCSV has a very short ORF AC4 with a coding capacity of only 47 amino acids. To see whether a longer AC4 protein would have an influence on virus symptoms and movement, we altered the AC4 stop codon into a codon for serine, the amino acid prevailing at this position in most AC4 proteins. The resulting WmCSV DNA-A mutant (WmCSV $\mathrm{AC} 4 * 48 \mathrm{~S}$ ) with an extended potential AC4 protein was not infectious by itself; coinoculation with DNA-B was still required for infectivity (Table 4). Also, no obvious effect on symptom severity and disease progression was caused by the WmCSV AC4*48S mutant. Concerning the role of AC4, WmCSV behaves similar to ACMV, in which truncation of an AC4 to 48 codons (16) or the complete knockout by suppression of its ATG codon had no effect on virus infectivity and Rep protein expression (22). However, the

TABLE 3. Agroinoculation of cloned Watermelon chlorotic stunt virus (WmCSV) DNA-A and DNA-B

\begin{tabular}{lccc}
\hline & \multicolumn{3}{c}{$\begin{array}{c}\text { No. of infected plants/no. of inoculated plants } \\
\text { (no. of experiments) }\end{array}$} \\
\cline { 2 - 4 } Plant species & DNA-A + DNA-B & DNA-A & DNA-B \\
\hline Watermelon & $248 / 250(5)$ & $0 / 15(3)$ & $0 / 15(3)$ \\
Nicotiana benthamiana & $499 / 500(5)$ & $0 / 20(3)$ & $0 / 20(3)$ \\
Melon & $4 / 4(1)$ & n.t. & n.t. \\
Tomato & $0 / 3(1)$ & n.t. & n.t. \\
\hline
\end{tabular}

${ }^{a}$ Agrobacterium tumefaciens containing WmCSV DNA-A and DNA-B inserted in the binary Ti-plasmid pBin19 were used as inoculum as described by Kheyr-Pour et al. (27). n.t. = Not tested. results of TGMV AL4 analyses are less conclusive, as independent genetic analyses mutating AL4 showed that it was not essential for virus replication and infectivity $(15,40)$. By contrast, its involvement in the regulation of AL1 (Rep protein) synthesis and in vitro expression were reported in other studies $(19,50)$.

Hence, unlike the essential function of $\mathrm{C} 4$ protein in the monopartite whitefly-transmitted geminiviruses (TYLCV and ToLCV) $(24,42)$, the biological role of a potential AC4/AL4 protein of bipartite geminiviruses including WmCSV still remains elusive.

An ORF AC5 protein is not required for infectivity. The sequence of WmCSV DNA-A revealed a potential ORF of complementary sense (AC5) with a coding capacity of 255 amino acids (Table 2). An equivalent ORF is also found in the genomes of several other whitefly-transmitted geminiviruses like ToLCV-In1/2 (39), TYLCVTh (43), ICMV (21), PHV (52), and AYVV (49). Although the expression of a protein encoded by ORF AC5 seemed unlikely, we nevertheless created three TGA termination codons at different positions in the coding sequence, altering codon R36, G76, and R146 into stop codons. The triple-stop mutation was introduced to prematurely terminate any potential protein synthesis beginning at the various ATG codons present in AC5. Agroinoculation of watermelon by the WmCSV AC5 triple-stop mutant led to symptoms indistinguishable from wild-type WmCSV (data not shown). Hence, ORF AC5 appears not to code for an essential protein.

Altering ORF BV2 of WmCSV-Sd has no influence on infectivity. Unlike DNA-B of WmCSV-Ir, DNA-B of WmCSV-Sd encodes an additional ORF with a coding capacity of 103 amino acids, preceding BV1 (Table 2). A frameshift mutation was introduced at codon 42 of ORF BV2 that caused a premature termination of a potential BV2 protein after a further 37 unrelated amino acids. The resulting WmCSV mutant was still infectious on watermelon, and no modifications of the disease symptoms were observed. This

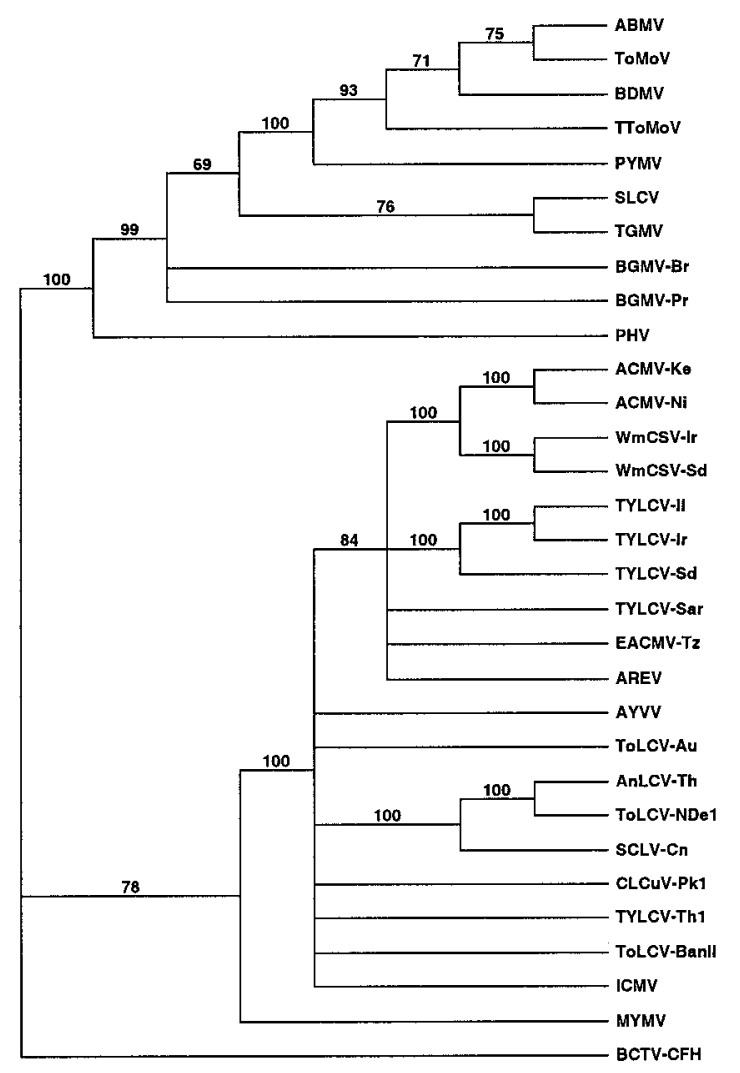

Fig. 4. Phylogenetic tree showing the relationships of selected begomoviruses. DNA-A sequences were aligned by Clustal W (51); tree construction and display was done using PAUP (D. L. Swofford, Illinois Natural History Survey, Champaign, IL). The sequence of Beet curly top virus (BCTV-CFH) was used as an outgroup. Virus names are according to Fauquet and Mayo (17). 
was consistent with the fact that DNA-B of the Iran isolate of WmCSV, the sequence of which was determined later during the analyses, lacked an ORF BV2. It was further corroborated by an experiment in which the B-components between the two WmCSV isolates were mutually exchanged prior to agroinoculation (reassortants). Disease symptoms of watermelon plants infected with DNAA of WmCSV-Sd and DNA-B of WmCSV-Ir and vice versa were indistinguishable from those of infections with the respective cognate DNA-A and DNA-B: 15 out of 15 plants inoculated for each combination showed the typical disease symptoms. These results provided further evidence that WmCSV-Sd and WmCSV-Ir are geographic variants of the same geminivirus species.

Identification of a whitefly-transmission determinant. The DNA-A clone of WmCSV from the Sudan was prepared from a sample that had been propagated by grafting for several months prior to DNA extraction. Despite the fact that the cloned viral DNA was fully infectious upon agroinoculation of watermelon, melon, and $N$. benthamiana, it was not transmissible by $B$. tabaci. In two independent experiments using 11 plants and a total of about 180 insects (16 per plant), the virions derived from cloned WmCSV DNA-A from the Sudan (WmCSV AV1-131D) turned out to be nontransmissible. A second set of three independent experiments using a total of 23 plants (15 insects per plant) inoculated by WmCSV AV1-131D and 11 plants inoculated by the engineered variant WmCSV AV1-D131N, employing a total of 345 and 165 insects, respectively, confirmed the transmissibility of WmCSV AV1-D131N. Seven plants out of eleven inoculated with WmCSV AV1-D131N developed disease, whereas all 23 plants assayed for transmissibility of WmCSV AV1-131D remained healthy. Hence, the alteration of capsid protein aspartate 131 into asparagine (D131N), an amino acid conserved at this position in all whitefly-transmitted geminiviruses, restored transmissibility of WmCSV.

Asparagine 131 is preceded by glutamine, of which the change into proline rendered TYLCV-Sar nontransmissible (36). Since N131 is part of a potential N-linked glycosylation site (NXS/T), it was tempting to speculate that capsid glycosylation might be required for successful whitefly transmission. However, no data on glycosylation of the capsid protein at N131 as a prerequisite for transmission are currently available. Curiously, mutations within this part of the genome appear to be frequent (36). Sequencing the capsid gene of another independent clone of WmCSV-Sd, derived directly from field-infected plants, revealed an asparagine codon at position 131 of the capsid gene, the sequence of the transmissible virus. Whether the nontransmissible WmCSV variant represented a rare or a more frequent member of the natural virus population or whether a relative accumulation of that particular mutant virus had been favored by its graft propagation prior to cloning cannot be determined because of the limited sample size analyzed.

A comparable result was reported for a transmissible isolate of ACMV (ACMV-NOg), in which repeated mechanical inoculation to $N$. benthamiana had led to the loss of whitefly transmissibility after 20 to 25 passages, while, when using the cloned ACMV-NOg genome for propagation, 40 passages did not alter its insect transmissibility. Therefore, the ACMV-NOg field isolates were prob-

TABLE 4. Appearance of viral DNA on watermelon after agroinoculation by DNA-A \pm DNA-B and DNA-A AC4*48S + DNA-B ${ }^{a}$

\begin{tabular}{lccc}
\hline & \multicolumn{3}{c}{ No. of infected plants/no. of inoculated plants } \\
\cline { 2 - 4 } Viral DNA & DNA-A + & DNA-A AC4*48S + & \\
detected after & DNA-B & DNA-B & DNA-A \\
\hline 5 days & $0 / 10$ & $0 / 10$ & $0 / 10$ \\
7 days & $9 / 10$ & $9 / 10$ & $0 / 10$ \\
9 days & $10 / 10$ & $10 / 10$ & $0 / 10$ \\
6 days & $10 / 10$ & $10 / 10$ & $0 / 10$ \\
\hline
\end{tabular}

${ }^{a}$ Agrobacterium tumefaciens containing WmCSV DNA-A and DNA-B inserted in the binary Ti-plasmid pBin19 were used as inoculum as described by Kheyr-Pour et al. (27). ably mixtures of insect-transmissible and -nontransmissible variants of the same virus (31).

Apart from the whitefly-transmission determinant described here and the one described by Noris et al. (36), five other amino acids in the N-terminal region of the capsid protein of AbMV were also reported as being potentially involved in transmission (55). However, no data are available on the respective individual importance of these amino acids for transmission.

Recently, the implication of a GroEL homologue from Buchnera sp. in the transmission by Bemisia spp. of TYLCV has been described (32). Hence, studying the interaction of geminivirus capsid with endosymbiont- and insect-encoded proteins may shed further light on the transmission process and could eventually provide a molecular rationale for a virus with an altered capsid being nontransmissible.

Given the wide distribution of watermelon chlorotic stunt viruses throughout the Near East and the endemic populations of $B$. tabaci in the Mediterranean Basin, particular attention should be given to this virus. Otherwise, it could invade cucurbit crops including melon in a similar way as the continuing devastation of tomato cultures by the Eastern Hemisphere tomato yellow leaf curl viruses within the Mediterranean Basin and their recent 'escape beyond' (47).

\section{ACKNOWLEDGMENTS}

This work was supported, in part, by grants from CNRS and INRA. K. B. thanks ICARDA for a doctoral fellowship. We thank D. Walkey for providing a polyclonal anti-WmCSV serum. We also thank D. Marian for technical assistance with the whiteflies and P. Capy for valuable assistance with PAUP.

\section{LITERATURE CITED}

1. Akbar Behjatnia, S. A., Dry, I. B., and Ali Rezaian, M. 1998. Identification of the replication-associated protein binding domain within the intergenic region of tomato leaf curl geminivirus. Nucleic Acids Res. 26: 925-931.

2. Argüello-Astorga, G. R., Guevara-Gonzalez, R. G., Herrera-Estrella, L. R., and Rivera-Bustamante, R. F. 1994. Geminivirus replication origins have a group-specific organization of iterative elements: A model for replication. Virology 203:90-100.

3. Ausubel, F. M., Brent, R., Kingston, R. E., Moore, D. D., Siedman, J. G., Smith, J. A., and Struhl, K. 1987. Current Protocols in Molecular Biology. Wiley, New York.

4. Bananej, K., Kheyr-Pour, A., and Ahoonmanesh, A. 1998. Identification of watermelon chlorotic stunt virus WCSV in Iran. Page 194 in: Proc. 13th Iranian Plant Prot. Congr., Karaj. Ministry of Agriculture, Tehran, Iran.

5. Bedford, I. D., Briddon, R. W., Jones, P., Alkaff, N., and Markham, P. G. 1994. Differentiation of three whitefly-transmitted geminiviruses from the Republic of Yemen. Eur. J. Plant Pathol. 100:243-257.

6. Bendahmane, M., Schalk, H.-J., and Gronenborn, B. 1995. Identification and characterization of wheat dwarf virus from France using a rapid method for geminivirus DNA preparation. Phytopathology 85:14491455.

7. Bevan, M. 1984. Binary Agrobacterium vectors for plant transformation. Nucleic Acids Res. 12:8711-8721.

8. Caciagli, P., Bosco, D., and Al-Bitar, L. 1995. Relationships of the Sardinian isolate of tomato yellow leaf curl geminivirus with its whitefly vector Bemisia tabaci Genn. Eur. J. Plant Pathol. 101:163170 .

9. Chatterji, A., Padidam, M., Beachy, R. N., and Fauquet, C. M. 1999. Identification of replication specificity determinants in two strains of tomato leaf curl virus from New Delhi. J. Virol. 73:54815489.

10. Choi, I. R., and Stenger, D. C. 1995. Strain-specific determinants of beet curly top geminivirus DNA replication. Virology 206:904-912.

11. Choi, I. R., and Stenger, D. C. 1996. The strain-specific cis-acting element of beet curly top geminivirus DNA replication maps to the directly repeated motif of the ori. Virology 226:122-126.

12. Dafalla, G. A., Gronenborn, B., Kheyr-Pour, A., and Lecoq, H. 1998. Watermelon chlorotic stunt virus: A new emerging epidemic in export melons in Sudan. Page 37 in: 2nd Int. Workshop Bemisia Geminiviruses, San Juan, Puerto Rico. 
13. Devereux, J., Haeberli, P., and Smithies, O. 1984. A comprehensive set of sequence analysis programs for the VAX. Nucleic Acids Res. 12:387-395.

14. Dry, I. B., Rigden, J. E., Krake, L. R., Mullineaux, P. M., and Rezaian, M. A. 1993. Nucleotide sequence and genome organization of tomato leaf curl geminivirus. J. Gen. Virol. 74:147-151.

15. Elmer, J. S., Brand, L., Sunter, G., Gardiner, W. E., Bisaro, D. M., and Rogers, S. G. 1988. Genetic analysis of the tomato golden mosaic virus. II. The product of the AL1 coding sequence is required for replication. Nucleic Acids Res. 16:7043-7060.

16. Etessami, P., Saunders, K., Watts, J., and Stanley, J. 1991. Mutational analysis of complementary-sense genes of African cassava mosaic virus DNA A. J. Gen. Virol. 72:1005-1012.

17. Fauquet, M. C., and Mayo, M. A. 1999. Abbreviations for plant virus names. Arch. Virol. 144:1249-1273.

18. Fontes, E. P., Eagle, P. A., Sipe, P. S., Luckow, V. A., and HanleyBowdoin, L. 1994. Interaction between a geminivirus replication protein and origin DNA is essential for viral replication. J. Biol. Chem. 269: 8459-8465.

19. Gröning, B. R., Hayes, R. J., and Buck, K. W. 1994. Simultaneous regulation of tomato golden mosaic virus coat protein and AL1 gene expression: Expression of the AL4 gene may contribute to suppression of the AL1 gene. J. Gen. Virol. 75:721-726.

20. Harrison, B. D. 1985. Advances in geminivirus research. Annu. Rev. Phytopathol. 23:55-82.

21. Hong, Y. G., Robinson, D. J., and Harrison, B. D. 1993. Nucleotide sequence evidence for the occurrence of three distinct whitefly-transmitted geminiviruses in cassava. J. Gen. Virol. 74:2437-2443.

22. Hong, Y., and Stanley, J. 1995. Regulation of African cassava mosaic virus complementary-sense gene expression by N-terminal sequences of the replication-associated protein AC1. J. Gen. Virol. 76:2415-2422.

23. Innis, M. A., Gelfant, D. H., Sninsky, J. J., and White, T. J. 1990. PCR Protocols: A Guide to Methods and Applications. Academic Press, San Diego, CA.

24. Jupin, I., De Kouchkovsky, F., Jouanneau, F., and Gronenborn, B. 1994. Movement of tomato yellow leaf curl geminivirus (TYLCV): Involvement of the protein encoded by ORF C4. Virology 204:82-90.

25. Jupin, I., Hericourt, F., Benz, B., and Gronenborn, B. 1995. DNA replication specificity of TYLCV geminivirus is mediated by the amino-terminal 116 amino acids of the Rep protein. FEBS (Fed. Eur. Biochem. Soc.) Lett. 362:116-120.

26. Kato, K., Onuki, M., Fuji, S., and Hanada, K. 1998. The first occurrence of tomato yellow leaf curl virus in tomato (Lycopersicon esculentum Mill.), in Japan. Phytopathol. Soc. Jpn. 64:552-559.

27. Kheyr-Pour, A., Bendahmane, M., Matzeit, V., Accotto, G. P., Crespi, S., and Gronenborn, B. 1991. Tomato yellow leaf curl virus from Sardinia is a whitefly-transmitted monopartite geminivirus. Nucleic Acids Res. 19: 6763-6769.

28. Laufs, J., Traut, W., Heyraud, F., Matzeit, V., Rogers, S. G., Schell, J., and Gronenborn, B. 1995. In vitro cleavage and joining at the viral origin of replication by the replication initiator protein of tomato yellow leaf curl virus. Proc. Natl. Acad. Sci. U.S.A. 92:3879-3883

29. Lazarowitz, S. G. 1992. Geminiviruses: Genome structure and gene function. Crit. Rev. Plant Sci. 11:327-349.

30. Lecoq, H., Dafalla, G. A., Mohamed, Y. F., Pitrat, M., Omara, S. E., and Ali, H. A. 1994. A survey on cucurbit viruses in Kordofan, east and central regions of Sudan. Khartoum Univ. J. of Agric. Sci. 1:47-71.

31. Liu, S., Bedford, I. D., Briddon, R. W., and Markham, P. G. 1997. Efficient whitefly transmission of African cassava mosaic geminivirus requires sequences from both genomic components. J. Gen. Virol. 78:1791-1794

32. Morin, S., Ghanim, M., Zeidan, M., Czosnek, H., Verbeek, M., and van den Heuvel, J. F. 1999. A GroEL homologue from endosymbiotic bacteria of the whitefly Bemisia tabaci is implicated in the circulative transmission of tomato yellow leaf curl virus. Virology 256:75-84.

33. Morris, B., Coates, L., Lowe, S., Richardson, K., and Eddy, P. 1990. Nucleotide sequence of the infectious cloned DNA components of African cassava mosaic virus (Nigerian strain). Nucleic Acids Res. 18:197-198.

34. Navot, N., Ber, R., and Czosnek, H. 1989. Rapid detection of tomato yellow leaf curl virus in squashes of plants and insect vectors. Phytopathology 79:562-568.

35. Navot, N., Pichersky, E., Zeidan, M., Zamir, D., and Czosnek, H. 1991. Tomato yellow leaf curl virus: A whitefly-transmitted geminivirus with a single genomic component. Virology 185:151-161.

36. Noris, E., Vaira, A. M., Caciagli, P., Masenga, V., Gronenborn, B., and Accotto, G. P. 1998. Amino acids in the capsid protein of tomato yellow leaf curl virus that are crucial for systemic infection, particle formation, and insect transmission. J. Virol. 72:10050-10057.

37. Orozco, B. M., and Hanley-Bowdoin, L. 1996. A DNA structure is required for geminivirus replication origin function. J. Virol. 70:148-158.

38. Padidam, M., Beachy, R. N., and Fauquet, C. M. 1995. Classification and identification of geminiviruses using sequence comparisons. J. Gen. Virol. 76:249-263

39. Padidam, M., Beachy, R. N., and Fauquet, C. M. 1995. Tomato leaf curl geminivirus from India has a bipartite genome and coat protein is not essential for infectivity. J. Gen. Virol. 76:25-35.

40. Pooma, W., and Petty, I. T. 1996. Tomato golden mosaic virus open reading frame AL4 is genetically distinct from its $\mathrm{C} 4$ analogue in monopartite geminiviruses. J. Gen. Virol. 77:1947-1951.

41. Pringle, C. R. 1998. The universal system of virus taxonomy of the International Committee on Virus Taxonomy (ICTV), including new proposals ratified since publication of the Sixth ICTV Report in 1995. Arch. Virol. 143:203-210

42. Rigden, J. E., Krake, L. R., Rezaian, M. A., and Dry, I. B. 1994. ORF C4 of tomato leaf curl geminivirus is a determinant of symptom severity. Virology 204:847-850.

43. Rochester, D. E., DePaulo, J. J., Fauquet, C. M., and Beachy, R. N. 1994. Complete nucleotide sequence of the geminivirus tomato yellow leaf curl virus, Thailand isolate. J. Gen. Virol. 75:477-485.

44. Rybicki, E. P. 1994. A phylogenetic and evolutionary justification for three genera of Geminiviridae. Arch. Virol. 139:49-77.

45. Rybicki, E. P., and Hughes, F. L. 1990. Detection and typing of maize streak virus and other distantly related geminiviruses of grasses by polymerase chain reaction amplification of a conserved viral sequence. $J$. Gen. Virol. 71:2519-2526.

46. Salati, R., Nakhla, M. K., Maxwell, D. P., Carvalho, M. G., Hou, Y. M., and Gilbertson, R. L. 1997. Complete nucleotide sequence of an infectious clone of tomato yellow leaf curl geminivirus from the Dominican Republic. (Abstr.) Phytopathology 87(suppl.):S84.

47. Simon Moffat, A. 1999. Geminiviruses emerge as serious crop threat. Science 286:1835.

48. Stanley, J., and Gay, M. R. 1983. Nucleotide sequence of cassava latent virus DNA. Nature 301:260-262.

49. Tan, P. H., Wong, S. M., Wu, M., Bedford, I. D., Saunders, K., and Stanley, J. 1995. Genome organization of ageratum yellow vein virus, a monopartite whitefly-transmitted geminivirus isolated from a common weed. J. Gen. Virol. 76:2915-2922.

50. Thömmes, P. A., and Buck, K. W. 1994. Synthesis of the tomato golden mosaic virus AL1, AL2, AL3 and AL4 proteins in vitro. J. Gen. Virol. 75:1827-1834

51. Thompson, J. D., Higgins, D. G., and Gibson, T. J. 1994. CLUSTAL W: Improving the sensitivity of progressive multiple sequence alignment through sequence weighting, position specific gap penalties and weight matrix choice. Nucleic Acids Res. 22:4673-4680.

52. Torres-Pacheco, I., Garzon-Tiznado, J. A., Herrera-Estrella, L., and Rivera-Bustamante, R. F. 1993. Complete nucleotide sequence of pepper huasteco virus: Analysis and comparison with bipartite geminiviruses. J. Gen. Virol. 74:2225-2231.

53. Tracy, T. E., and Mulcahy, L. S. 1991. A simple method for direct automated sequencing of PCR fragments. Biotechniques 11:68-75.

54. Walkey, D. G. A. 1992. Plant virus diseases of Yemen and associated areas. Overseas Development Administration (ODA) Publ., London.

55. Wu, Z. C., Hu, J. S., Polston, J. E., Ullman, D. E., and Hiebert, E. 1996. Complete nucleotide sequence of a nonvector-transmissible strain of Abutilon mosaic geminivirus in Hawaii. Phytopathology 86:608-613.

56. Zhou, X., Robinson, D. J., and Harrison, B. D. 1998. Types of variation in DNA-A among isolates of East African cassava mosaic virus from Kenya, Malawi and Tanzania. J. Gen. Virol. 79:2835-2840. 